\title{
HOW CEREAL GRASS SHOOTS PERCEIVE AND RESPOND TO GRAVITY ${ }^{1}$
}

\author{
Peter B. Kaufman, Thomas G. Brock, Il Song, \\ Young BoK Rho, ${ }^{2}$ AND NAJATI S. GHOSHEH ${ }^{3}$ \\ Department of Biology, University of Michigan, Ann Arbor, Michigan 48109
}

\begin{abstract}
A BS T R A C T
The leaf-sheath pulvinus of grasses presents a unique system for studying gravitropism, primarily because of its differences from other organs. The mature pulvinus is a discrete organ specialized for gravitropism: it is nongrowing in the absence of gravistimulation and capable of displaying a graviresponse independent of the rest of the plant. In this paper we present a model for gravitropism in pulvini based on recent findings from studies on the mechanisms of graviperception and graviresponse. According to this model, amyloplasts play an essential role in perceiving a change in the orientation of the pulvinus. The perception of this reorientation leads to the enhanced synthesis and release from conjugate of the auxin IAA, and the increased conjugation of gibberellin, on a localized basis. Because there is a graded growth promotion across the gravistimulated pulvinus, it is suggested that the observed hormonal asymmetry is actually an indication of a linear gradient of hormone concentration, as well as hormone response, across the pulvinus. It is further suggested that the linear gradient of hormone concentration may be predominantly the result of local changes in hormone level, rather than a product of hormonal movement into or across the pulvinus.
\end{abstract}

All GRASS SHOOTs have distinctive regions that function primarily as graviresponsive organs. These swollen regions near the sites of leaf insertion have been variously termed nodes (Wright, 1986), pseudopulvini (Salisbury and Ross, 1985) or pulvini (Brown, Pratt, and Mobley, 1959; Pharis et al., 1981). We prefer the term pulvinus (plural, pulvini), since this structure is a specialized mature organ that is physically distinct from the node (i.e., it is located distal to the point of insertion of the leaf on the stem), and because its specialization as a motor unit is analogous to the classical pulvinus (although the mechanism of action is quite different). This pulvinus actually repre-

\footnotetext{
I Received for publication 18 December 1986; revision accepted 24 March 1987.

2 Department of Biology, Chosun University, Kwangju, Korea.

${ }^{3}$ Department of Biology, Eastern Michigan University, Ypsilanti, Michigan 48197.

We thank Robert Bandurski, Jerry Cohen, P. Dayanandan, C. I. Franklin, M. L. Hsieh, Roger O'Neill, Daphne Osborne, Lisa Parker, Dick Pharis, Barbara Pickard, Frank Salisbury, Tom Scott, Paul Thompson and Malcolm Wilkins for helpful discussions in connection with the preparation of this manuscript. We also express our thanks and appreciation to Thora Halstead, Chief of NASA's Space Biology Program, for her encouragement and for financial support under NASA grant NAGW-34. Also supported by a NASA Research Associate Award to TGB and by President Chyll Woong Park of Chosun University to YBR.

Abbreviations: $\mathrm{GA}_{3}=$ gibberellic acid; $\mathrm{IAA}=$ indole3-acetic acid.
}

sents either a swollen region above the intercalary meristem at the base of an internode (in panicoid grasses) or a swollen leaf sheath base (in festucoid grasses) (Fig. 1). The pulvinus functions in controlling the orientation of the vegetative shoot, and ultimately that of the inflorescence, via asymmetric growth within the pulvinus, returning the shoot to the vertical in response to lodging or gravistimulation. Lodging refers to the prostration of grass shoots, often resulting from strong wind and/or rain. Gravistimulation refers to the displacement of the shoot in any position away from the vertical; in research, this typically involves placing the shoot in a horizontal position, perpendicular to the force of gravity on earth.

Both the recovery from lodging and the selfcorrecting of slightly angled shoots to vertical positions by means of asymmetric growth in the pulvini of grasses are adaptively significant for several reasons: 1) both result in the orientation of photosynthetic organs (stems, leaves and young inflorescences) to maximize primary productivity while minimizing water loss; 2) the former aids in pollen dispersal by wind when plants are in anthesis, thus insuring greater reproductive success for the plants; and 3) the former also increases the number of shoots which can develop to reproductive maturity within a given area. If cereal grass shoots fail to recover from lodging, due to the presence 
of heavy, grain-filled inflorescences, serious losses in grain yield can result.

The grass pulvinus (Fig. 2-4), as a graviresponsive system, offers many distinct advantages for studying the mechanism of gravitropic responses in plants. These include: 1) the pulvinus is a relatively large mass of tissue, so relatively few samples are needed for the analysis of changes in metabolites or hormones during gravistimulation; 2) the sites for gravity perception and response are in close proximity within the single organ, obviating problems associated with long-distance hormone transport; 3) a long lag time exists between stimulus and response (minimum of 15 min; Kaufman and Dayanandan, 1984), permitting convenience in studying the earliest events that occur in the upward bending response (compare with a lag time of 1.5 to $3 \mathrm{~min}$ for corn coleoptiles (Bandurski et al., 1984)); 4) the system can rerespond to repeated gravistimulations, in contrast to roots and dicot stems, in which the graviresponding zone changes with plant growth; 5) there exist "lazy" mutants of some grasses, which are agravitropic (i.e., they show no graviresponse), allowing the study of the nature of the lesion in the gravitropic mechanism and therefore the identification of critical components of the mechanism.

The primary objective of this paper is to review what is known about the upward bending response in grass shoot pulvini. While the gravity perception-transduction-response system of pulvini is in many ways comparable to that found in other organs, some differences exist and these will be considered. Also, directions for future work regarding the roles of starch grains, calcium, macromolecules (such as cellulose, pectins, structural proteins, wallloosening enzymes and calmodulin) and hormones in the pulvinus graviresponse will be presented.

GENERAL FEATURES OF GRAVITROPISM IN GRASS SHOOT PULVINI - When intact cereal grass shoots become lodged or are gravistimulated, they begin to show an upward bending response within $30 \mathrm{~min}$, which continues over a comparatively long period of 60 to $72 \mathrm{hr}$. This type of gravitropic response is termed negative gravitropic curvature, and it results in shoots being oriented to a vertical position (Fig. 5). The gravitropic response is the manifestation of asymmetric growth within the pulvinus.

The pattern of growth within the pulvinus is different from that in other shoots and in roots. In shoots (e.g., sunflower hypocotyl; Hart and MacDonald, 1984) and in roots (e.g., maize; Barlow and Rathfelder, 1985), asymmetric

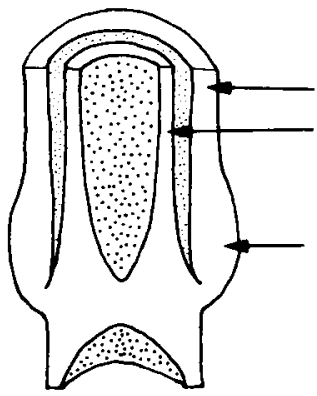

A

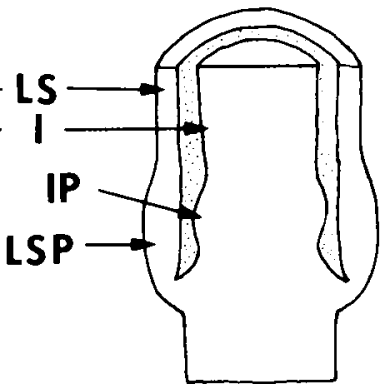

B
Fig. 1. Diagrammatic representation of a longitudinal bisection of the pulvinus region of festucoid $(A)$ and panicoid (B) grasses. Festucoid grasses have a leaf-sheath pulvinus (LSP) at the base of the leaf sheath (LS). Panicoid grasses have a prominent internodal pulvinus (IP) at the base of the internode (I); some panicoid grasses also have a leaf-sheath pulvinus.

growth results from the promotion of growth on one side of the organ. Frequently, a reduction or even cessation of growth is observed on the opposite side. In these cases, the graviresponse can be described as distinctly lateral, with asymmetric form resulting primarily from unilateral growth promotion. Growth promotion in shoots is commonly found to be the product of the reinitiation of growth in an area that had ceased to elongate (e.g., Hart and MacDonald, 1984). In contrast, mature, competent pulvini display almost no elongation growth before lodging or gravistimulation (Arslan and Bennet-Clark, 1960). Asymmetric growth in pulvini, in response to horizontal placement, results from growth promotion throughout the organ, with the response increasing in a linear fashion from the top side to the bottom side (Dayanandan and Kaufman, 1984). Hence, the gravistimulation of pulvini results in graded growth promotion. The graviresponse is also a promotion of growth in a region that had previously not been elongating.

Does upward bending in the pulvinus involve any compression of cells on the uppermost side of a graviresponding pulvinus? Figures 6,7 , and 8 provide an answer to this question. These scanning electron micrographs were prepared from similar areas of critical point-dried pulvini, from a vertical barley shoot (Fig. 6), the top side of a pulvinus of a gravistimulated barley shoot ( $24 \mathrm{hr}$ treatment, 30 degrees curvature, Fig. 7), and the bottom side of the same gravistimulated pulvinus (Fig. 8). There is no significant compression of epidermal cells on the top side. However, cortical cells internal to the epidermis have become compressed along their periclinal walls, while 


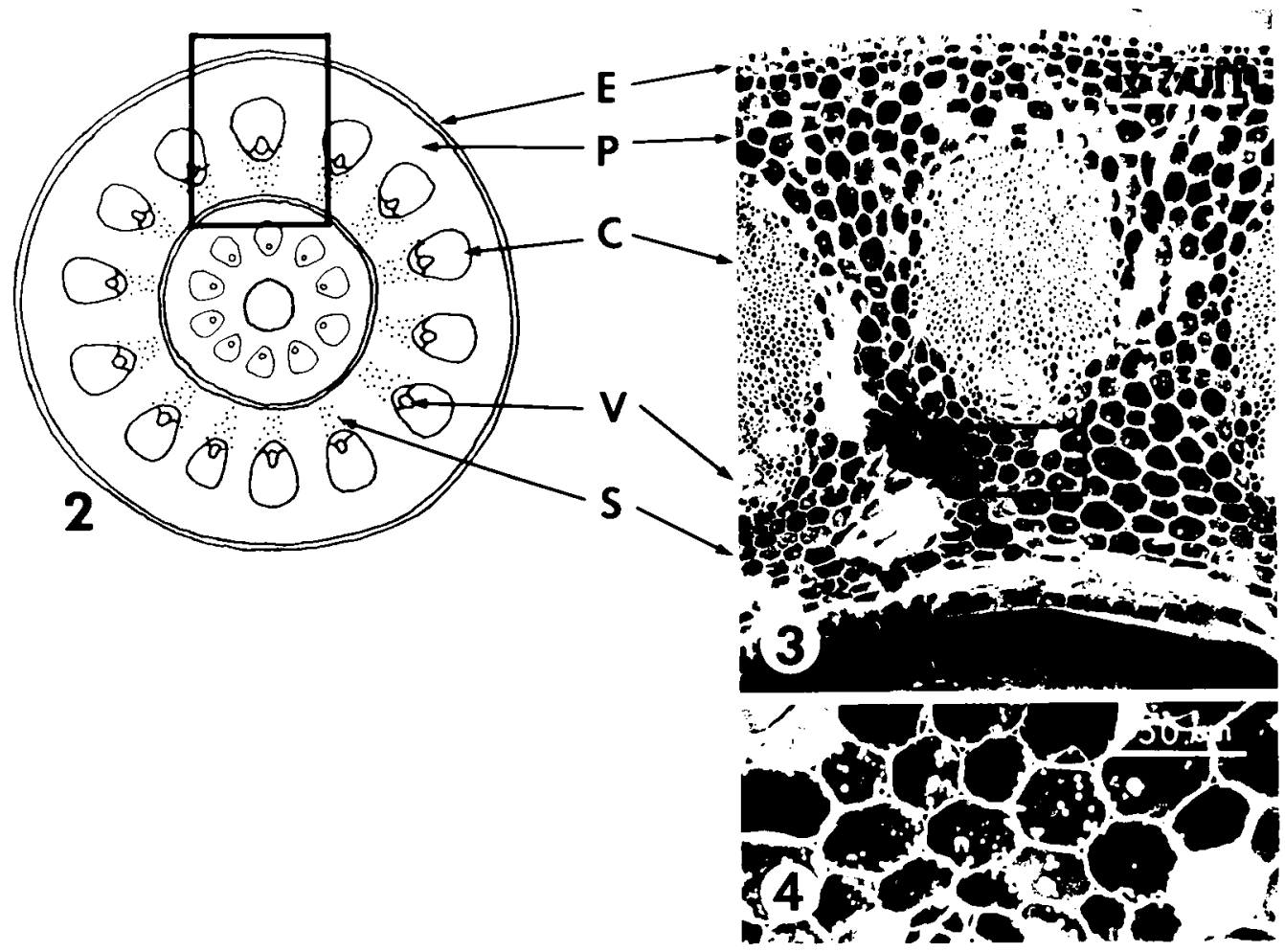

Fig. 2-4. 2. Cross-sectional diagram, depicting the midportion of a barley leaf-sheath pulvinus and internode base enclosed within it. $\mathrm{E}=$ epidermis, $\mathrm{P}=$ parenchyma tissue, $\mathrm{C}=$ collenchymatous tissue associated with each vascular bundle, $\mathrm{V}=$ vascular bundle, $\mathrm{S}=$ statenchyma tissue. 3. Scanning electron micrograph prepared from a cross section of a barley leaf-sheath pulvinus, comparable to the enclosed region in Fig. 2. 4. Scanning electron micrograph showing the statenchyma tissue (comparable to the enclosed area in Fig. 3). Starch statoliths appear as small white granules in each statocyte and are noticeably smaller than the nucleus (n).

the anticlinal walls are comparable in length to those in the vertical cortex. As a result, the cortical cells in the uppermost portion of gravistimulated pulvini can have as little as half the volume of equivalent cells in upright, control pulvini. Compression can occur, but it is seen primarily in cells of the inner cell layers below the epidermal system at the top side of a graviresponding pulvinus. In no case have we ever observed any decrease in length of the pulvinus on the top side of one which has responded by bending upward. It is comparable in length to the lateral flank of an upright pulvinus, so no compression takes place at this locus in contrast to what occurs in subjacent cell layers. This property may result from differences in cell wall rigidity and plasticity between cells of the epidermal system and those of the cortical tissue below.

The kinetics of upward bending in pulvini is different from that in other organs. Barley (Hordeum vulgare cv. Larker) and oat (Avena sativa $\mathrm{cv}$. Victory) pulvini start to bend upward after a lag time of at least $15 \mathrm{~min}$.
Features of the response of oat pulvini are presented in Fig. 9 and Table 1. The kinetics of this response were determined through the use of an angular recording position transducer (Kaufman and Dayanandan, 1984). For this purpose, an excised shoot was mounted with the basal end in $0.1 \mathrm{M}$ sucrose and left in a vertical orientation in the dark for $0.5 \mathrm{hr}$. The mounted section was then rotated to a horizontal position and the trainsducer arm was placed on the leaf sheath portion beyond the pulvinus, and left in the dark at $25 \mathrm{C}$. Excised shoot portions containing pulvini were from next-to-last nodes of 42- to 45-day-old oat plants. We find that continuous upward bending in the pulvinus of an excised shoot portion under the above conditions begins at least 20 min after gravistimulation in oat pulvini. Similarly treated barley pulvini show a lag time before the upward bending response of at least $15 \mathrm{~min}$. Upward bending then progresses in linear fashion at 4 degrees $\mathrm{hr}^{-1}$ for barley and 1.5 degrees $\mathrm{hr}^{-1}$ in oats for the next $12 \mathrm{hr}$, then gradually decelerates over the next $60 \mathrm{hr}$. No 


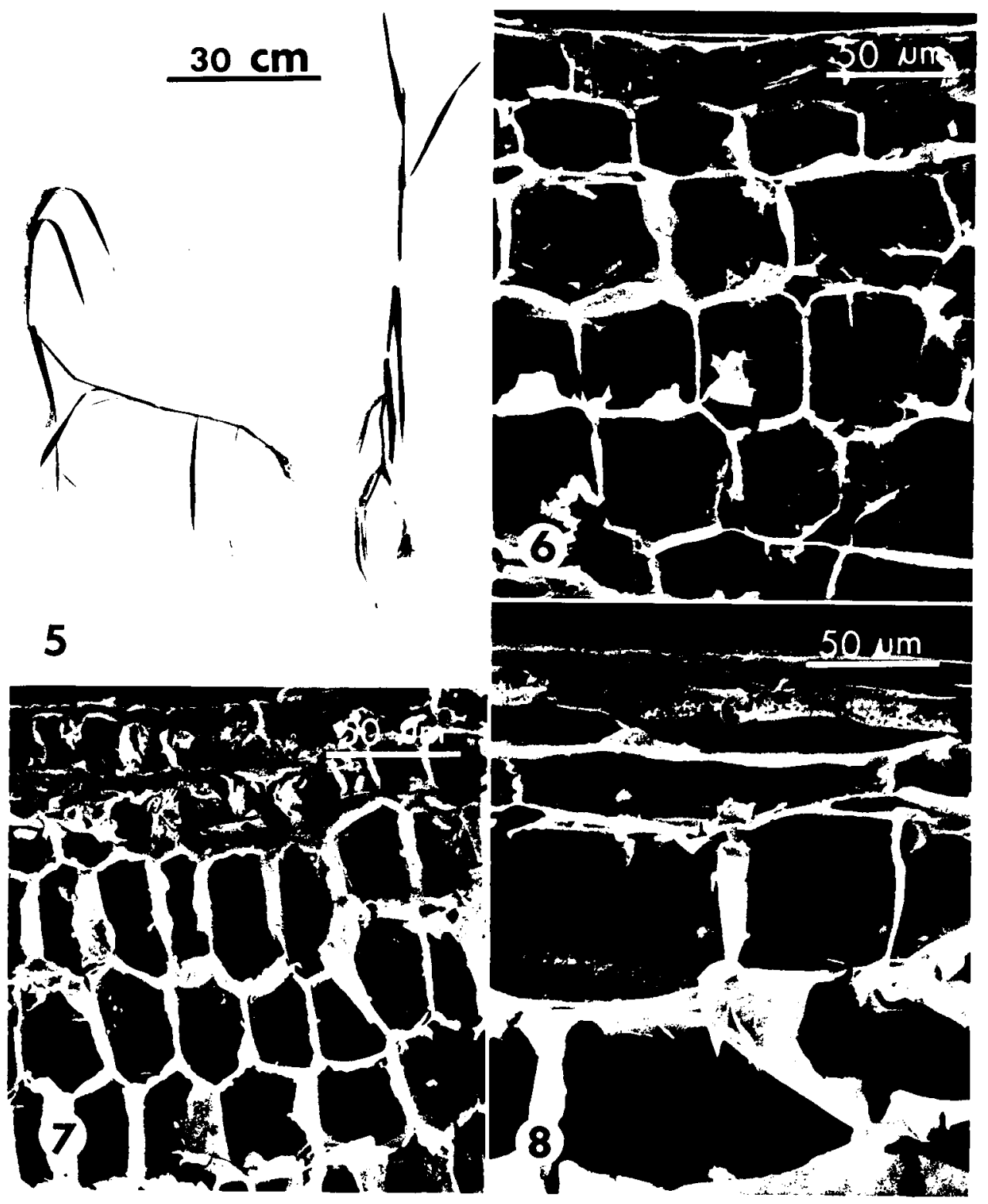

Fig. 5-8. 5. The graviresponse in lodged (left) and erect (right) plants of cultivated oat (Avena sativa L., cv. Victory). In the lodged plant, two pulvini have responded to bring the shoot to a vertical orientation. In the erect plant, note the "self-correcting" of plant orientation by distinct graviresponses of the pulvini. 6-8. Scanning electron micrographs of longitudinal sections of pulvini of barley, epidermis at top. 6. Epidermal and cortical regions from a pulvinus in a vertically-oriented shoot. 7. "Top" side of a gravistimulated pulvinus after $24 \mathrm{hr}$. Note the reduction in length of periclinal cell walls in the cortex. 8. "Bottom" side of the same gravistimulated pulvinus.

further bending is observed after $72 \mathrm{hr}$. In contrast, in maize coleoptiles (Zea mays cv. Stowells Evergreen), the upward bending response in horizontally positioned plants is much faster; namely, 1 to 2 degrees min $^{-1}$ over a 60 - to 90 -min period (Bandurski et al., 1984). With maize seedlings and with excised barley and oat shoots, angles of bending of 90 degrees are typically obtained. Such angles of curvature are seldom seen in pulvini of intact barley and oat plants which have been lodged or gravistimulated since several pulvini are involved in righting the shoots to a vertical position.

A unique feature of pulvini is the capacity of the graviresponding region to re-respond to subsequent gravistimulations. In dicot shoots and in roots, the graviresponding region "moves" with time as the organ grows, and so 


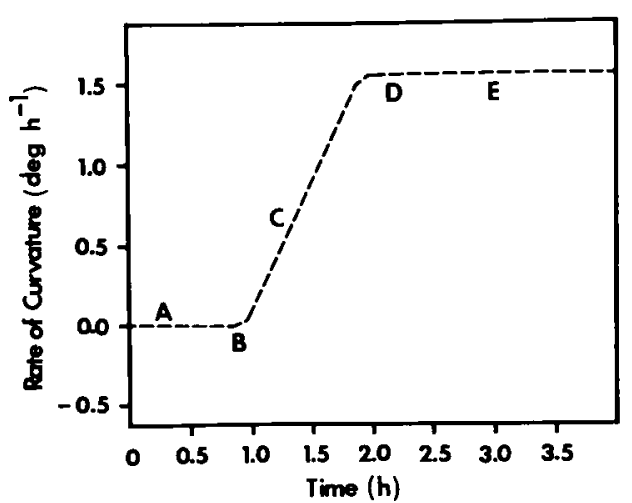

Fig. 9. Generalized summary of the early kinetics of the graviresponse in oat leaf-sheath pulvini. Data for the earliest response (A), time of initial upward bending response (B), half time to steady curvature rate (C), overshoot of response (D) and final steady curvature rate (E) are summarized in Table 1.

new cells are involved in subsequent graviresponses. When pulvini in excised shoot portions of intact plants are rotated 180 degrees after a 30-degree upward bending response has occurred, the pulvini reverse their direction of curvature through further stimulation of growth across a new gradient. This response requires about 25 to $30 \mathrm{~min}$ after the shoots are first rotated (Brock and Kaufman, unpublished results). Successive rotations can be made as many as five times, and reversal of curvature can be elicited in each case, though the time required to achieve equivalent angles of curvature increases with each new rotation. Figure 10 shows the final outcome of five successive re-stimulations by rotation in a pulvinus from an intact barley shoot. Note that the pulvinus has elongated considerably as a result of these successive rotations and repeated upward bending responses. Never have we observed

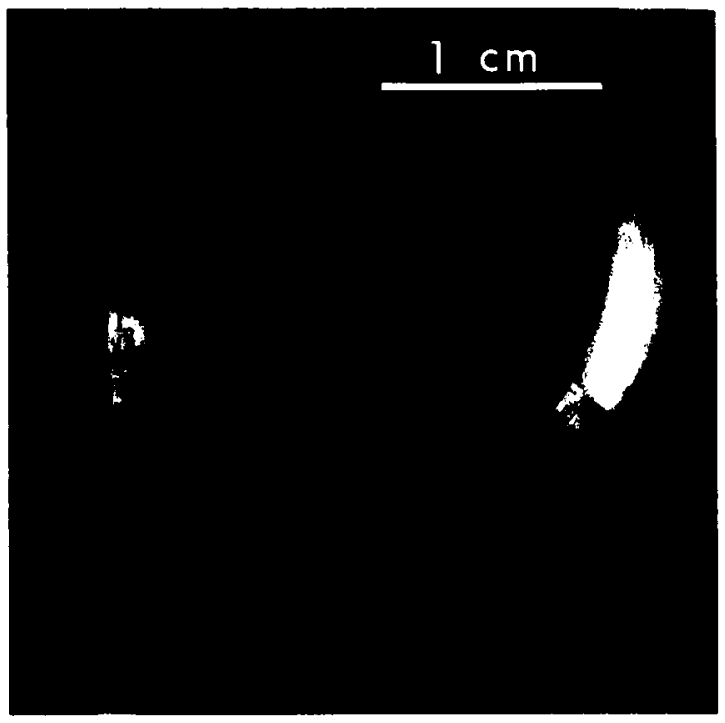

Fig. 10. Pulvini from barley plants left erect (left) or repeatedly gravistimulated (right). The pulvinus can rerespond to changes in orientation. The final result is extreme elongation of the pulvinus.

such an elongation response in upright pulvini. Similarly, treatment of pulvini in upright plants with hormones such as IAA or GAs cause only a small growth response. However, brassinosteroid, especially in the presence of IAA, will cause extensive pulvinus elongation in upright barley shoots (Kaufman, Brock, and Song, in press). In other words, the pulvinus consists of unelongated mature cells which only elongate in response to gravistimulation or lodging. The re-response may have some adaptive significance in that lodged shoots which do start to bend upwards may become lodged again or fall over again due to the weight of a developing inflorescence. In either case, gravitropically

TABLE 1. Features of the graviresponse of the oat leaf-sheath pulvinus in excised segments, determined by continuous monitoring with an angular displacement transducer. Segments were excised $10 \mathrm{~cm}$ below and $4 \mathrm{~cm}$ above the $(p-1$ locus) pulvinus, mounted with the base in $0.1 \mathrm{M}$ sucrose, and left in a vertical orientation in the dark, $25 \mathrm{C}$, for 30 min before gravistimulation. $\mathrm{N}=50$
(A)
Initial response:
Significant transient upward bending
No significant initial bending
Significant transient downward bending
percentage:
8
27
65
(B) Lag time $(\mathrm{min})$ to continuous upward bending: Mean: 58.1 ( $\mathrm{SE}=21)$ minimum: 20
maximum: 125
(C) Half time $(\mathrm{min})$ to a steady continuous upward bending rate: Mean: $27.3(\mathrm{SE}=13) \quad$ minimum: 10
maximum: 64
(D) Percent observed to significantly overshoot the subsequent steady bending rate:
(E) Final steady upward bending rate $\left(\operatorname{deg~} \mathrm{hr}^{-1}\right)$ : Mean: $1.5(\mathrm{SE}=0.46) \quad$ minimum: 0.60 
TABLE 2. The effect of alpha-amylase, with or without subsequent sucrose treatment, on the graviresponse of barley pulvini. Plants were treated in a vertical (V) position with alpha-amylase (hog pancrease; Sigma Co.), then sucrose $(S u c ; 0.1 \mathrm{M})$, and then gravistimulated $(G)$. Each treatment was for $24 \mathrm{hr}$. Six samples per treatment, repeated three times

\begin{tabular}{lcc}
\hline \hline & $\begin{array}{c}\text { Mean graviresponse } \\
\text { (degrees) }\end{array}$ & $\begin{array}{c}\text { Percent control } \\
\text { graviresponse }\end{array}$ \\
\hline Water $(\mathrm{V}) \rightarrow$ Water $(\mathrm{G})$ & 38.5 & $(100)$ \\
$3.13 \mathrm{mg} / \mathrm{ml}$ amylase $(\mathrm{V}) \rightarrow$ Water $(\mathrm{G})$ & 5.5 & 14.3 \\
$6.25 \mathrm{mg} / \mathrm{ml}$ amylase $(\mathrm{V}) \rightarrow$ Water $(\mathrm{G})$ & 0 & 0 \\
Water $(\mathrm{V}) \rightarrow$ Suc $(\mathrm{V}) \rightarrow$ Suc $(\mathrm{G})$ & 62.4 & $(100)$ \\
$3.13 \mathrm{mg} / \mathrm{ml}$ amylase $(\mathrm{V}) \rightarrow$ Suc $(\mathrm{V}) \rightarrow$ Suc $(\mathrm{G})$ & 54.5 & 87.3 \\
$6.25 \mathrm{mg} / \mathrm{ml}$ amylase $(\mathrm{V}) \rightarrow$ Suc $(\mathrm{V}) \rightarrow$ Suc $(\mathrm{G})$ & 29.9 & 47.9 \\
\hline
\end{tabular}

competent pulvini on these shoots can re-respond to the new gravitropic stimulus.

The capacity for a given pulvinus to respond to a lodging or gravistimulation event is a function of the state of development of the pulvinus. In order for a pulvinus to respond to gravistimulation, it must be relatively ligninand silica-free, and it must have graviperceptive organelles (starch statoliths) present (Dayanandan and Kaufman, 1984; Wright, 1986). Increased silicification has been correlated with the loss of the capacity of Equisetum shoots to respond to gravistimulation (Srinivasan, Dayanandan, and Kaufman, 1979). Thus, it appears that in older pulvini, where the walls of the cells have become lignified and silicified, no bending response occurs when these pulvini are gravistimulated. Likewise, young pulvini which have not yet developed starch statoliths or have too few present do not respond to gravistimulation (Wright, 1986; also, see next section). Within a given plant, the lodging or gravistimulatory event is perceived and responded to by several pulvini. In large grasses such as sugarcane, maize, sorghum and bamboo, as many as six to eight pulvini are involved in the upward bending response, whereas in cereal grasses such as oats, rice, wheat, rye, and barley, it involves a maximum of four pulvini. These cereal grasses may also respond gravitropically at any time during the course of vegetative shoot development whenever the shoots become oriented slightly away from the vertical position. They respond by making small "corrections" in pulvinus angle by asymmetric growth that results in orienting the shoot to a vertical position. These features are indicated in Fig. 5.

Perception of GRAVITY IN THE PULVINUSThe first step in the graviresponse is the perception of a change in orientation with respect to the force of gravity. The evidence indicates that certain cells ("statocytes") are specialized for this purpose. These cells typically contain starch-filled plastids ("amyloplasts"), which move in response to changes in the orientation of the cell. Historically, this sedimentation of the amyloplasts (or "statoliths") has been assumed to be the first step in the graviresponse (Haberlandt, 1900; Nemec, 1900). Although several lines of evidence support this assumption, a recent study of the roots of a starch-free form of Arabidopsis, which display a graviresponse comparable to that of starch-containing forms, indicates that amyloplasts are not essential for graviperception in roots (B. Pickard, personal communciation). Still, it is not clear whether amyloplasts would function in some graviperception capacity in roots, when present.

There is incontrovertible evidence that amyloplasts are indeed the graviperceptive organelles in cereal grass pulvini. Two lines of evidence support this view. First, when the starch in the amyloplasts in statocytes (Fig. 11) of Larker barley is lysed through treatment with alpha-amylase for $24 \mathrm{hr}$ (Fig. 12), graviresponse in the pulvinus is nullified (Table 2 ). Then, when the starch in these organelles is resynthesized following treatment with $0.1 \mathrm{M}$ sucrose for another $24 \mathrm{hr}$ (Fig. 13), the pulvini respond to gravistimulation. Second, young pulvini of wild oats (Avena fatua) which are developing new statocytes do not respond to gravistimulation until they have formed 14 to 16 statoliths per statocyte, and as this number increases to 25 statoliths per statocyte, there is a parallel, linear increase in angle of bend over a given time interval in the pulvinus (Wright, 1986). Alpha-amylase is a fairly large protein with molecular weight of $62-63 \mathrm{Kd}$; it is assumed that, in these experiments, it may be actively taken up through intact cellular membranes, or, alternatively, it could reach the pulvinus statenchyma cells symplastically, via plasmodesmata.) In the face of this evidence, we cannot accept the idea that these starch-containing plastids in grass pulvini have nothing to do with graviperception. 


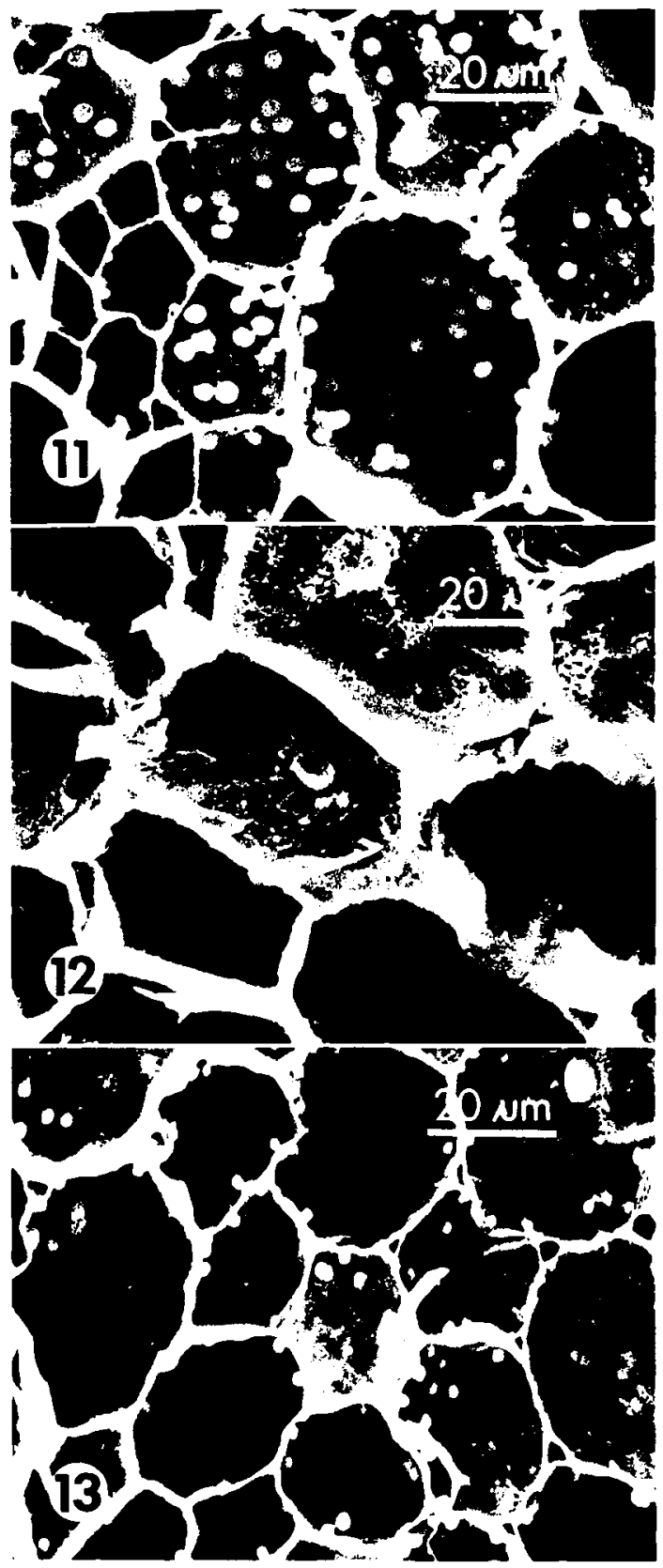

Fig. 11-13. Scanning electron micrographs of barley pulvini statocytes. 11. Statocytes of untreated pulvini. Note the presence of numerous, large amyloplasts. 12. Statocytes of pulvini after treatment with $6.25 \mathrm{mg} / \mathrm{ml}$ hog pancreas alpha-amylase for $24 \mathrm{hr}$ in dark. Note absence of amyloplasts. 13. Statocytes treated for $24 \mathrm{hr}$ with $6.25 \mathrm{mg} / \mathrm{ml}$ alpha-amylase, then for $24 \mathrm{hr}$ with $0.1 \mathrm{~m}$ sucrose.

Now we can ask, how might the starch statoliths function in graviperception in the grass pulvinus system? In the gravistimulated pulvini of Larker barley and of Victory oats, the

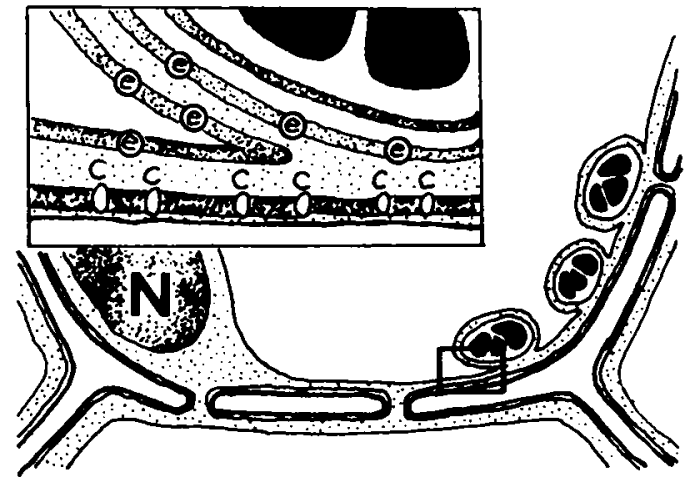

Fig. 14. Diagrammatic representation of a cross section of a statocyte shortly after gravistimulation, showing nucleus $(\mathrm{N})$ and three sedimenting amyloplasts (at right). As the amyloplasts fall, they bring enzymes (" $e$ " in inset), bound to the amyloplast and tonoplast membranes, to interact with components of the plasmalemma of the cell's lateral face. It is proposed that the plasmalemma contains IAA-deconjugating and gibberellin-conjugating moieties (" $C$ " in inset), which are activated by amyloplast sedimentation to release bound forms of IAA and bind-free gibberellin. The gaps in the cell wall in the diagram represent plasmodesmata.

starch statoliths complete their descent within two min (Thompson, Song, and Kaufman, unpublished results). This is well within the perception times required for a graviresponse to occur in the pulvini of these plants. When the statoliths descend, they impinge upon the large vacuole within each statocyte. In doing so, they bring down a variety of membranes with them, including the double-layered plastid membrane and, in many cases, portions of the tonoplast membrane (Kaufman and Dayanandan, 1984; see also micrographs in Parker, 1979). We have proposed that the starch statoliths in cereal grass pulvini may serve as "information carriers" (Kaufman and Dayanandan, 1984), by bringing membrane-bound enzymes to sites where IAA can be released from a (presumedly) bound, conjugated form, or gibberellins can be actively conjugated (Fig. 14). The free IAA may then directly initiate asymmetric growth on a local basis, or move laterally and/or downward within the pulvinus. Hence, enzymes such as esterases and peptidases, which hydrolyze, respectively, myoinositol ester IAA and amidelinked IAA, and glucosyl transferases, which direct the formation of glucosyl esters of GAs from the free GA form, would be activated as a result of amyloplast sedimentation.

The ideas that statoliths directly alter enzyme activity and that this mediates the gravity response are highly speculative and will require further testing in order to prove or disprove their validity. To test these ideas, one might 
start with enzyme localization on the statocyte membranes, or in a gross fashion, on the whole statocytes. We have made an initial attempt in this direction with the statocytes of Victory oats. If fresh pulvinus tissue preparations are stained with fluorescein diacetate, which is specific for esterase activity, the statocytes fluoresce bright yellow when excited by UV light in the microscope (Dayanandan and Kaufman, unpublished results). The fluorescence is largely confined to the statoliths. Such esterase activity associated with the starch statoliths could be involved in deconjugating IAA from its myoinositol ester conjugate that we have postulated to be localized in the plasma membrane.

It is possible that the starch statoliths perform yet another function; namely, to act as "pressure probes" that would open or activate ion channels in the plasma membrane by a process called "stretch activation" (B. Pickard, personal communication). This idea presupposes that the portions of membranes that contain ion channels (e.g., for $\mathrm{Ca}^{2+}, \mathrm{K}^{+}, \mathrm{H}^{+}$, or $\mathrm{Mg}^{2+}$ ) are attached to a network of microtubules in the cytosol. This network would be placed under increased tension as statoliths impinge on them at the bottom of the cell. Changes in the tension on the microtubules would bring about a change in the conductance of ion channels in the plasma membrane. The end result of the stretching process would be an altered rate of ion flux, theoretically leading to activation of cellular processes. The movement of $\mathrm{Ca}^{2+}$ and its association with calmodulin action have been implicated in the gravitropic response of roots (e.g., Hasenstein and Evans, 1986; Moore and Evans, 1986), suggesting that calcium channels might be significant in the stretch activation process. However, as far as we are aware, nothing is known about the roles of calcium and calmodulin in the gravitropic response mechanism in cereal grass pulvini.

The significance of both pressure-sensitive ion channels and calcium in graviperception in pulvini are key areas for future research. The "stretch activation" idea might be tested through the use of patch clamping techniques applied to statocyte protoplasts in order to measure membrane potential changes associated with cells in which the starch grains sediment in comparison with cells in which the starch grains are free-floating and do not sediment. Fluxes of ions such as $\mathrm{Ca}^{2+}$ into and out of statocytes could be indicated in upright pulvini as compared with gravistimulated pulvini by measuring extracellular calcium levels with a $\mathrm{Ca}^{2+}$-specific microelectrode. Unfortunately, quantitation of cytosolic calcium re- mains technically difficult. In particular, attempts to use some of the new fluorescent $\mathrm{Ca}^{2+}$ indicators, such as Fura-2 or Quin-2, have met with considerable difficulty when used on plant cells (P. Hepler, personal communication).

In summary, the way that gravity is perceived by plants is complex. Apparently, more than one mechanism may be involved. These different mechanisms may function independently or they may interact. For example, pressure-sensitive channels in cell membranes may be capable of responding to changes in gravity independent of starch grain sedimentation, but their response may be amplified or prolonged following sedimentation. Similarly, the amyloplasts may have multiple functions. By moving in response to cell reorientation, they may transport membrane-bound information, reorient microtubules or alter local membrane properties. Also, the starch may serve as a substrate source for energy production, cell wall synthesis or osmotic pressure maintenance during growth. Many of these ideas are only now being addressed experimentally.

TRANSDUCTION OF THE GRAVISTIMULUS - The classical model of gravitropism in coleoptiles and roots designates the site of perception as the tip of the organ, with the response occurring at a site near the elongation zone some millimeters away. Simply because of the separation of sites, some mode of transduction of the signal from tip to growth zone must exist. The nature of this transduction process remains unknown. Among the more exciting possibilities are electrical potential differences generated across the stimulated organ (Behrens, Gradmann, and Sievers, 1985; T. Bjorkman and A. C. Leopold, personal communication) and induced asymmetry of metal ions, such as calcium (Slocum and Roux, 1983; Hasenstein and Evans, 1986).

The cereal pulvinus lacks the obvious separation of sites for perception and response (Fig. 2-4). The transduction of signal need only be over a few cells, from the statocytes to the site of growth regulation. In the pulvinus, the primary site of cell expansion appears to be the collenchyma tissue, which is adjacent to the vascular system and near the statoliths (Dayanandan, Hebard, and Kaufman, 1976; Dayanandan et al., 1977; also see Fig. 2-4). The epidermis is not considered to be essential for the graviresponse, since preliminary tests find only a minor effect of epidermal removal on the change in angle of curvature over time, in response to gravistimulation (Dayanandan and Kaufman, unpublished results). Due to the short distance between the statoliths and col- 
lenchyma, the nature of the transduction process in pulvini may be very different from that in other organs. Also because of the short distance between these sites, this aspect of the graviresponse in pulvini may be the most difficult to solve. At present, there is nothing known about the transduction process in grass shoot pulvini.

RESPONSE TO GRAVISTIMULATION: HORMONAL CHANGES-Ultimately, the graviresponse of plants is a growth response. The reorientation of the plant following lodging or gravistimulation is the product of asymmetric growth. From the earliest publications of studies on tropisms came the postulation that the response is directly due to an asymmetric distribution of growth regulators, such as hormones (Cholodny, 1926; Went, 1926). This concept has persisted and remains an underlying assumption of much of the research in gravitropism today.

Such a simple concept has still not achieved universal support, however. A number of investigators (Digby and Firn, 1976; Firn and Digby, 1980, and references cited therein; Mertens and Weiler, 1983) have suggested that asymmetric distributions of hormones may not direct the upward bending response in gravistimulated shoots. Rather, they say that differences in tissue sensitivity to hormones may account for the differential growth response. Furthermore, the timing of the occurrence of the asymmetry in hormonal distribution is a central issue. These investigations argue that hormone asymmetries may develop slowly and may in fact be the result of the graviresponse.

While the question of tissue sensitivity has yet to be addressed, recent studies using grass seedlings and shoot pulvini have dealt with the issue of timing. In gravistimulated maize seedlings, greater amounts of auxin, both as free IAA and as the myoinositol ester of IAA, can be found on the lower side (relative to upper) in as short a time as five min (R. Bandurski, personal communication). This coincides with the time when an upward bending response can first be measured (Bandurski et al., 1984). Similarly, in the graviresponse of leaf-sheath pulvini of wild oat (Avena fatua), free IAA asymmetry has been measured in as early as $15 \mathrm{~min}$ after the shoots are first gravistimulated (Wright, 1986, and papers cited therein). This is at least five min before the gravistimulated shoots start to bend upward. Consistent with these findings is the report that an asymmetry of IAA is observable in gravistimulated Echinochloa colonum nodes (Wright, Mousdale, and Osborne, 1978).
On the other hand, ethylene asymmetry in gravistimulated cultivated oats (Avena sativa) arises as late as $5.5 \mathrm{hr}$ after upward bending is initiated in gravistimulated plants (Kaufman et al., 1985). For this reason, it is apparent that ethylene is not one of the primary regulatory hormones in eliciting the upward bending response in pulvini of these plants. Its asymmetry may arise as a result of the unequal distribution of free IAA in the upward bending pulvinus, especially since it is well-documented that ethylene synthesis can be promoted by IAA. (See Kaufman et al., in press, and references cited therein.) Thus, we have a situation in which some hormones, such as ethylene, are not involved in regulating the initiation of upward bending, and other hormones, such as IAA, which may be directly involved. Auxin is therefore one of the leading candidates for causing the upward bending response, since its asymmetry arises at or just before the upward curvature is initiated.

It should be noted that the asymmetry of a hormone such as IAA need not be large in order to initiate an upward bending response. This is the case with IAA in maize seedlings. A very small gradient in IAA across a tissue can elicit considerable unequal growth of the tissue, certainly enough for the initial growth asymmetry seen in graviresponding tissue, since the growth rate of a section is the sum of the growth rates of a series of individual cells. Also, when one adds up these gradients across a whole pulvinus, for example, the expected effects on differential cell elongation would be quite significant. Thus, it is seen that very small differences in IAA concentration can elicit a rather significant differential growth response, especially since IAA can stimualte cell elongation at concentrations as low as $10^{-9} \mathrm{M}$ in roots and $10^{-7}$ $M$ in shoots (Thimann, 1977). One cannot, of course, rule out differential sensitivity to IAA as an integral component of the unequal growth response mechanism.

Gibberellins (GAs) may also be involved in gravitropism in pulvini. During gravistimulation, glucosyl ester conjugates of GAs accumulate in the top halves whereas free GAs $\left(\mathrm{GA}_{4}, \mathrm{GA}_{7}, \mathrm{GA}_{1}\right)$ accumulate in the bottom halves; further, feeds with ${ }^{3} \mathrm{H}-\mathrm{GA}_{4}$ indicate that there is differential synthesis of free $G_{1}$ and $\mathrm{GA}_{7}$ and $\mathrm{GA}$ conjugates at the two positions, with more free GAs being synthesized in the bottom halves and more GA conjugates being synthesized in the upper halves (Pharis et al., 1981; Kaufman and Dayanandan, 1984). In sum, it appears that both differential synthesis and release from conjugate are involved in the establishment of hormone asymmetry in gravi- 
responding cereal grass pulvini. In order to resolve when this occurs, kinetic studies must be undertaken to determine relative amounts of both free IAA and its conjugates and free GAs and their conjugates in top and bottom halves of graviresponding pulvini.

An important question we can now ask is this: how do hormone asymmetries arise? Basipolar transport (i.e., lateral transport in horizontally-oriented segments) is unlikely for IAA (Kaufman et al., 1979), since there is no significant downward movement of ${ }^{14} \mathrm{C}$-IAA applied in agar blocks to "top" sides of gravistimulated pulvini in cultivated oats (Avena sativa) and wheat (Triticum aestivum) (Bridges and Wilkins, 1973). The same can be said for ${ }^{3} \mathrm{H}$ $\mathrm{GA}_{3}$, applied in the same fashion to gravistimulated oat pulvini (Kaufman and Dayanandan, 1984). We are then left with three other possibilities: 1) differential hormone metabolism, 2) differential hormone synthesis, and 3) release of free hormone from conjugate. Current evidence favors the last two as being most important. For IAA, in gravistimulated oat pulvini which have achieved angles of bending equal to $30^{\circ}$ in a 24 -hr period, we have found seven times more free IAA in the gravistimulated pulvini than in those of the upright controls, and the asymmetry is ca. 1:2.5 top/bottom in the former (Table 3). As Wright (1986) has pointed out, this IAA asymmetry arises as early as $15 \mathrm{~min}$ after the pulvini are placed horizontally. It appears then that differential IAA synthesis can account for at least some of this asymmetry.

More recently, Kaufman and Cohen (1986, unpublished results) have found that the upright control oat pulvinus contains three times more amide- plus ester-linked IAA than free IAA, suggesting that IAA is stored in the pulvinus as its conjugate. Further, we suggest that it could be released differentially from this conjugate during gravistimulation. This idea is currently being tested. It is a fact that in pulvini in an upright position, ${ }^{14} \mathrm{C}$-IAA can move from top to bottom in the expected basipolar fashion, although much of this IAA does not exit the pulvinus at its base into the internode below, so it appears not to be a "leaky" system. That which does move into the pulvinus from shoot parts above it (leaves, inflorescence), then, most likely gets impounded as IAA conjugate, which in vegetative oat tissues is primarily amide-linked IAA (Bandurski and Schulze, 1977).

This model for gravitropism, as applied to the grass pulvinus, calls for a basic departure from the classical model of gravitropism, as applied to coleoptiles (Cholodny, 1926; Went,
TABLE 3. Analysis of free IAA amounts in vertical and gravistimulated oat pulvini. Plants were gravistimulated intact for $24 \mathrm{hr}$. Pulvini (10 g fresh weight total per value) were then excised and assayed for free $I A A$ content by the double isotope dilution technique (Bandurski and Schulze, 1977)

\begin{tabular}{cc}
\hline & $\begin{array}{c}\text { Free IAA } \\
\text { (ng/g dry weight) }\end{array}$ \\
\hline Vertical pulvinus & 70 \\
Gravistimulated pulvinus & \\
Upper half & 130 \\
Lower half & 350 \\
\hline
\end{tabular}

1926) or roots (e.g., Shaw and Wilkins, 1973; Pilet, 1977). According to the classical model, hormones function in the transduction phase of gravitropism: perception at one site leads to response at another through asymmetric basipetal transport of hormones. In the pulvinus, basipetal transport of hormones is not a factor during gravitropism: the pulvinus can fully perceive and respond to gravistimulation as an isolated organ (Brock and Kaufman, unpublished results). Moreover, it appears that basipolar transport (i.e., downward transport in horizontally-placed pulvini) is relatively insignificant as a transduction mechanism. In pulvini, a key response to gravistimulation is a change in the rates of synthesis and deconjugation of IAA and conjugation of GA. Asymmetric growth is assumed to be a direct effect of the local change in hormone level. In this situation, transduction becomes, simply, the events between amyloplast sedimentation anc change in hormone level.

According to this model, then, a variety of molecular and cellular changes, related to hormone action and growth promotion, should be observable. This is indeed the case. Analysis of tissue from vertical vs. gravistimulated barley pulvini, by sodium dodecyl sulfate-polyacrylamide gel electrophoresis, indicates that several protein levels change in response to gravistimulation (Kaufman, Song, and Pharis, 1985). Specific enzyme assays indicate an asymmetry in the activity of beta-glucosidase, cellulase and invertase in response to gravistimulation (Kaufman, Song, and Pharis, 1985). The syntheses of RNA and proteins are required for the gravitropic response, since actinomycin D and cycloheximide completely inhibit the asymmetric growth response (Dayanandan, Franklin, and Kaufman, 1982). Work in progress is designed to determine if these changes in protein levels are exactly the changes expected if gravitropism is purely the result of differential hormone action.

In summary, the response phase of pulvinus 
gravitropism may involve auxin, gibberellin(s) and ethylene (Kaufman and Song, 1987). Auxin is dramatically increased by both de novo synthesis and release from conjugate. An asymmetry in auxin distribution can be measured in $15 \mathrm{~min}$, which is before the initial upward bending is observed. Gibberellins also are found to change in response to a gravistimulus. There is enhanced conjugation of GAs in upper halves of pulvini, resulting in an asymmetry of free, active gibberellin. Also, ethylene levels increase, but only after $5.5 \mathrm{hr}$ of gravistimulation. Hence, ethylene does not promote upward bending, but may play a role in later events. An important point is that asymmetry of auxin and gibberellin levels is not due to transport into or across the responding organ, but is most likely the product of local, cellular processes. Also, changes in tissue sensitivity upon gravistimulation have yet to be addressed in detail, although recent studies on dicot hypocotyls suggest that a change in sensitivity to endogenous auxin may be a fundamental feature of the graviresponse (Salisbury et al., 1986; Meudt, 1987). It is possible that this is also an important facet of the graviresponse mechanism in cereal grass shoots.

Gravitropism IN THE PULVINUS IN COMPARISON WITH OTHER SYSTEMS-Gravitropism in the grass pulvinus is similar in many ways to gravitropism in other organs. First, starch grains, as components of amyloplasts, are associated with the perception of a change in the orientation of the organ with respect to the force of gravity. In the pulvinus, the starch grains are a necessary component for perception: upon enzymatic removal of the starch, no response occurs; after reformation, the competence to respond returns. Second, the graviresponse can be directly attributed to an asymmetry of hormones in an active form. A dramatic increase in free IAA occurs and a graded asymmetry is observed before asymmetric growth is measured.

Several distinct differences place the pulvinus apart from other graviresponse systems. First, the site of perception is very close to the site of response. This may allow for unique mechanisms of transduction and response. Second, the organ is capable of repeated graviresponses within one defined region. This reresponse feature implies the involvement of changes in tissue sensitivity and suggests several possible avenues for future research. Third, the graviresponse is under multiple hormonal control. Both gibberellins and auxin are involved in the initiation of asymmetric growth, and ethylene may affect later stages of the re- sponse. Finally, hormonal transport, either into or across the organ, appears to be relatively unimportant in the initiation of the graviresponse. Hormonal asymmetries result chiefly through local processes, such as enhanced synthesis and a shift between the free and conjugated forms of the hormone.

\section{LITERATURE CITED}

Arslan, N., and T. A. Bennet-Clark. 1960. Geotropic behaviour of grass nodes. J. Exp. Bot. 11: 1-12.

Bandurski, R. S., AND A. Schulze. 1977. Concentrations of indole-3-acetic acid and its derivatives in plants. Pl. Physiol. 60: 211-213.

,-- , P. Dayanandan, and P. B. Kaufman. 1984. Response to gravity by Zea mays seedlings. I. Time course of the response. Pl. Physiol. 74: 284288.

Barlow, P. W., and E. L. Rathfelder. 1985. Distribution of extension growth along vertical and horizontal gravireacting maize roots. Planta 165: 134 141 .

Behrens, H. M., D. Gradmann, and A. Sievers. 1985. Membrane-potential responses following gravistimulation in roots of Lepidium sativum L. Planta 163: $463-472$.

Bridges, I. G., AND M. B. Wilkins. 1973. Growth initiation in the geotropic response of wheat node. Planta 112: 191-200.

Brown, W. V., G. A. Pratt, and H. M. Mobley. 1959. Grass morphology and systematics. II. The nodal pulvinus. Southwest. Naturalist 4: 126-130.

Cholodny, N. 1926. Beitrage zur Analyse der geotropischen Reaktion. Jahrb. wiss. Bot. 65: 447-459.

Dayanandan, P., C. I. Franklin, and P. B. Kaufman. 1982. Linkage between gravity perception and response in the grass leaf-sheath pulvinus. The Physiol. 25: $101-102$.

$\longrightarrow$, H. V. Hebard, V. D. Baldwin, and P. B. KaufMAN. 1977. Structure of gravity-sensitive sheath and internodal pulvini in grass shoots. Amer. J. Bot. 64: 1189-1199.

$\longrightarrow$, AND P. B. Kaufman. 1976. Cell elongation in the grass pulvinus in response to geotropic stimulation and auxin application. Planta 131: 245252.

nificance of gravity-induced asymmetric growth in the grass leaf-sheath pulvinus. Ann. Bot. 53: 29-44.

DigBY, J., AND R. D. FIRN. 1976. A critical assessment of the Cholodny-Went theory of shoot gravitropism. Curr. Adv. Pl. Sci. 8: 953-960.

FIRN, R. D., AND J. DigBy. 1980. The establishment of tropic curvature in plants. Ann. Rev. Pl. Physiol. 31: 131-148.

Haberlandt, G. 1900. Über die Perzeption des geotropischen Reizes. Ber. Dtsch. Bot. Ges. 18: 261-272.

HART, J. W., AND I. R. MACDONAld. 1984. Is there a role for the apex in shoot geotropism? PI. Physiol. 74: 272-277.

Hasenstein, K.-H., And M. L. Evans. 1986. Calcium dependence of rapid auxin action in maize roots. $\mathrm{Pl}$. Physiol. 81: 439-443.

Kaufman, P. B., and P. Dayanandan. 1984. Hormonal regulation of the gravitropic response in pulvini of grass shoots. In S. S. Purohit [ed.], Hormonal regu- 
lation of plant growth and development, Vol. I, pp. 369-385. Agro Botanical Publishers, Bikaner, India. , AND I. SONG. 1987. Hormones and the orientation of growth. In P. J. Davies [ed.], Plant hormones and their role in plant growth and development, pp. 375-392. Martinus Nijhoff, Dordrecht, The Netherlands.

-, T. G. Brock, AND I. SoNG. In press. Chemical growth regulators. In F. C. Steward and A. D. Krikorian [eds.], Treatise on plant physiology. Academic Press, New York.

- I. SONG, AND R. P. Pharis. 1985. Gravity perception and response mechanism in graviresponding cereal grass shoots. In S. S. Purohit [ed.], Hormonal regulation of plant growth and development, Vol. II, pp. 189-200. Agro Botanical Publishers, Bikaner, India.

, R. P. Pharis, D. M. Reid, AND F. D. Beall. 1985. Investigations into the possible regulation of negative gravitropic curvatures in intact Avena sativa plants and in isolated stem segments by ethylene and gibberellin. Physiol. Pl. 65: 237-244.

- R. Bandurski, P. Dayanandan, R. Koning, AND M. HARRISON. 1979. Hormonal regulation of the negative geotropic response in Avena shoots. Pl. Physiol. 63: 143.

Mertens, R., AND E. W. Weiler. 1983. Kinetic studies on the redistribution of endogenous growth regulators in gravireacting plant organs. Planta 158: 339-348.

MEUDT, W. J. 1987. Investigations on the mechanism of the brassinosteroid response. Effect of brassinolide on gravitropism of bean hypocotyls. P1. Physiol. 83: 195-198.

Moore, R., ANd M. L. Evans. 1986. How roots perceive and respond to gravity. Amer. J. Bot. 73: 574-587.

Nemec, B. 1900. Uber die Art der Wahrnehmung des Schwerkraftreizes bei den Pflanzen. Ber. dtsch. Bot. Ges. 18: 241-245.

PARKer, M. L. 1979. Morphology and ultrastructure of the gravity-sensitive leaf sheath base of the grass $E \mathrm{ch}$ inochloa colonum L. Planta 145: 471-477.
Pharis, R. P., R. L. Legge, M. Noma, P. B. Kaufman, N. S. Ghosheh, J. D. LACroiX, AND K. Heller. 1981. Changes in endogenous gibberellins and the metabolism of ${ }^{3} \mathrm{H}-\mathrm{GA}_{4}$ after geostimulation in shoots of the oat plant (Avena sativa). Pl. Physiol. 67: 892-897.

PlLet, P. E. 1977. Growth inhibitors in growing and geostimulated maize roots. In P. E. Pilet [ed.], Plant growth regulation, pp. 115-128. Springer-Verlag, Berlin.

Salisbury, F. B., AND C. W. Ross. 1985. Plant physiology. 3d ed. Wadsworth, Belmont, CA.

-, P. Rorabaugh, R. White, and L. Gillespie. 1986. A key role for sensitivity to auxin in gravitropic stem bending. Pl. Physiol. 80: (S) 26.

Shaw, S., AND M. B. Wilkins. 1973. The source and lateral transport of growth inhibitors in geotropically stimulated roots of Zea mays and Pisum sativum. Planta 109: 11-26.

Slocum, R. D., And S. J. Roux. 1983. Cellular and subcellular localization of calcium in gravistimulated oat coleoptiles and its possible significance in the establishment of tropic curvature. Planta 157: 481-492.

Srinivasan, J., P. Dayanandan, and P. B. Kaufman. 1979. Silica distribution in Equisetum hyemale var. Affine L. (Engelm.) in relation to the negative geotropic response. New Phytol. 83: 623-626.

ThImanN, K. V. 1977. Hormone action in the whole life of plants. University of Massachusetts Press, Amherst, MA.

WeNT, F. W. 1926. On growth accelerating substances in the coleoptile of Avena sativa. Proc. K. Akad. Wet. Amsterdam 30: 10-18.

WRIGHT, M. 1986. The acquisition of gravisensitivity during the development of nodes of Avena fatua. J. Pl. Growth Regul. 5: 37-47.

, D. M. A. Mousdale, and D. J. Osborne, 1978. Evidence for a gravity-regulated level of endogenous auxin controlling cell elongation and ethylene production during geotropic bending in grass nodes. Biochem. Physiol. Pflanzen. 172: 581-596. 